\title{
On the use of Lateral Wave for the Interlayer Debonding Detecting in an Asphalt Airport Pavement using a Multi-static GPR System
}

\author{
Lilong Zou, Member, IEEE, Li Yi, Member, IEEE, and Motoyuki Sato, Fellow, IEEE
}

\begin{abstract}
In this paper, we focus on the detection of the interlayer debonding of the asphalt airport pavement by the Groundpenetrating Radar (GPR) system. Since the interlayer debonding usually occurs in the shallow region of the asphalt airport pavement (several centimeters), it is difficult to interpret the anomalies or the defects from the GPR signals composed of many waves under the boundary conditions. Moreover, the wavelength of the ordinary GPR system is over several centimeters. Therefore, the spatial resolution of the system is not accurate enough to consider the millimeter thickness of the debonding layer. To overcome these problems, we propose a new method based on evaluating the lateral wave behavior of common midpoint (CMP) gathers collected by a multiple static GPR system. The multi-static GPR system is a stepped frequency continuous wave (SFCW) radar system, which consists of 8 transmitting and 8 receiving bowtie antennas. The system operates in the frequency range from 50 MHz to $1.5 \mathrm{GHz}$. After the validation of the simulation, the results of the interlayer debonding detection were evaluated by a field experiment obtained at Tokyo International Airport. The proposed method can detect the debonding layers which are less than $1 \mathrm{~mm}$. Also, it is shown that our proposed method has a high consistency with the conventional acoustic finding method in the field measurement. It provides an innovative and effective method for the interlayer debonding detection of a partially damaged airport asphalt pavement, which is difficult to be observed by the ordinary GPR signals.
\end{abstract}

Index Terms-Ground-penetrating Radar (GPR), multi-static GPR system, lateral wave, common mid-point (CMP), asphalt airport pavement, nondestructive inspection, interlayer debonding detection.

\section{INTRODUCTION}

$\mathbf{T}$ HE maintenance of the airport pavement structure has presented many unique challenges. The airport engineering and the maintenance personnel must provide all-weather services to millions of people every year while maintaining millions of cubic meters of concrete distributed throughout the facilities. An accurate, inexpensive and efficient technology is needed to detect the innumerable internal anomalies in the concrete structures to ensure integrity and safety as well as meeting the original design specifications [1]. Because the airport pavement must be constructed with high standards,

Manuscript received xxxx; revised xxxx; accepted xxxx. This work was supported by xxxx. (Corresponding author: Lilong Zou).

L. Zou is with the School of Computing and Engineering, University of West London, London, W5 5RF, U.K. (e-mail: lilong.zou@uwl.ac.uk).

L. Yi is with the Graduate School of Engineering Science, Osaka University, Osaka 560-8531, Japan (e-mail: yi@ee.es.osaka-u.ac.jp).

M. Sato is with the Center for Northeast Asian Studies, Tohoku University, Sendai, 980-8576, Japan (e-mail: motoyuki.sato.b3@tohoku.ac.jp). including the perfect smoothness, toughness, and uniformity, it is necessary to use multiple layers of different materials [2]. However, such multi-layer structure is not enough to prevent all the problems. High temperatures and pressures generated by the moving aircraft on the airport pavement can cause slight raindrops entering the shallow areas of the pavement. These small amounts of water stay at the tiny spaces between the different layers. With the temperature changing of the shallow pavement structure, these small amounts of water evaporate into the vapor and extend to the thin cracks. When these thin cracks extend enough to affect the integrity of the road surface, the road surface may collapse, leading to the distortion or collapse of the road surface [1]-[3]. Therefore, it is important to identify these potentially damaged parts in advance and repair them before they collapse or bubble up suddenly. Hence, the detecting of interlayer debonding in the pavement mechanism has a significant impact on the remaining life of the pavement and the safety of the aircraft running in the airport. In a word, the early detection of the interlayer debonding is a basic problem in the airport pavement maintenance.

At present, there are only a limited number of technologies to inspect this concrete structure. These technologies are accurate and economical from integrity and safety as well as meeting the original design specifications. Remote sensing, non-destructive testing techniques such as the infrared thermal imaging, the ground-penetrating radar (GPR), the magnetometer and tachometer, are used to measure the physical properties affected by various materials and conditions in or under the concrete infrastructure. These techniques have established a reputation for investigating accurately the concrete anomalies [4][5]. Currently, the traditional methods to find these anomalous pavements are the acoustic finding and the infrared irradiation method by the skilled workers. Unfortunately, this kind of work is inefficient and has a high economic cost.

Among these different methods, it is well known that GPR has the best resolution and is widely used in civil engineering applications [6]-[11]. However, due to the limitation of the penetration depth and resolution, GPR is mainly used to detect the large-scale voids or distortions in the underground inspection [6][7]. The main difficulty of the data processing is to detect the minor anomalies from the backscattered signals in the airport pavement inspection. It is very different from the most road inspection applications, which focus on the largescale defects under the pavement at the meters depth. However, the interlayer debonding between the asphalt layers has a 
millimeter-scale width. Due to the limitation of the resolution, these thin layers (the interlayer debonding) of one-tenth or tens of wavelengths are difficult to detect by the radar signals. Therefore, we almost could not observe directly the responses of the thin cracks, as described in [12]-[14]. In addition, the minor anomalies or defects on the airport pavement occur in the shallow areas (several centimeters depth). Various waves generated under the boundary conditions are mixed with the responses from the small shallow anomalies, which makes it difficult to interpret the anomalies or defects by the GPR signals with a few $\mathrm{GHz}$ central frequency.

Because of the thin debonding layer in the pavement structure, the change of the density can be used as an index of the pavement inspection which has been achieved by various methods. Nuclear densitometers and ultrasonic measurements have excellent accuracy in the laboratory experiments, while they are not suitable for the large-scale inspection [15]-[17]. Infrared tomography is more suitable for large-scale inspection, but it is affected greatly by the observation conditions, which makes it difficult to be used in the practical application [18] [19]. Based on the same idea, the estimate of the dielectric constant or the variation of the propagation velocity of the electromagnetic wave can be used as an important index for the GPR detection of the underground anomalies [20][23]. However, in the traditional GPR profiles, the dielectric constant changes caused by the anomalies in the shallow areas of the pavement structure do not produce obvious reflectivity anomalies (see the example of Tokyo International Airport below). At the same time, due to the relatively low resolution of the GPR system and the difficulty of the parameter adjustment, it is hard to be used in real large-scale applications.

In [24], we have reported a novel approach using the interferometry technique to analyze two common midpoints (CMP) gather and find out the small deviations of the asphalt layers depth and the velocity changes for the airport pavement inspection. In this paper, we describe a new method for detecting the interlayer debonding directly in the pavement structures by analyzing the lateral wave characters of CMP gathers. Lateral wave is an electromagnetic wave generated at or near the plane boundary between two different dielectrics, such as the air and soil or the air and pavement surface [25]. The lateral wave propagates in the shallow subsurface region and suffers some attenuation, while propagates in the air without any attenuation over a long distance and finally arrives at the receiver [26]. Therefore, the lateral wave carries the information of the shallow subsurface region, which can be used to detect small anomalies in the shallow pavement structure. Based on this property, this paper presents a method to analyze the trend of the maximum amplitude of the lateral wave with the offset in CMP gathers after amplitude compensation. The linear function of the maximum amplitude and the antenna offset is estimated by the least square method. Finally, the gradient variation of the maximum amplitude with the antenna offset is obtained. By processing the real experimental data obtained from the taxiway pavement at Tokyo International Airport, the results obtained by the proposed method show excellent performance in detecting the interlayer debonding of the upper asphalt layer. The results also show a high-level consistent with the traditional acoustic finding methods.

The rest of the paper is followed by four sections. Section II describes the multi-static GPR system and the airport asphalt pavement structures. This section also describes how to arrange the measurement lines to extract a large number of CMP gathers from the multi-static GPR systems. By considering the properties of the asphalt pavement and the specific application of the airport asphalt pavement inspection, section III presents the corresponding theories, assumptions, and the considerations for the lateral wave analysis. Section IV discusses the validation of the proposed approach by the numerical simulation and the field experiment results. This section also shows the results of on-site coring verification at the airport. Section V summarizes the paper.

\section{Multi-STatic GPR System And Airport PaVement STRUCTURE}

\section{A. Multi-static GPR System}

In order to investigate the large-scale three-dimensional (3D) GPR measurements, we developed a multi-static ground penetrating radar system, shown in Fig. 1. It is a steppedfrequency radar system consisting of eight transmitting antennas and eight receiving antennas. The system works in the frequency range from $50 \mathrm{MHz}$ to $1.5 \mathrm{GHz}$ and can provide enough penetration with a good resolution for the ordinary shallow measurement [27]. Unlike the traditional impulse radar, each receiving antenna in this system collects data in the frequency domain.

Operators can manually pull the system and obtain the GPR data. The data acquisition is triggered at a sampling interval of $1 \mathrm{~cm}$ by a distance-measuring wheel. The system acquires a full 64-channel data, including all the combinations of the transmitters and receivers. By pushing the handlebars or pulling them with ropes, the system can scan up to $7 \mathrm{~km} / \mathrm{h}$. Through the single scan measurement, the system can cover a scanning area of $2 \mathrm{~m}$ wide. It is easy and fast to reconstruct the subsurface 3-D image by the acquired high-density GPR data [28]. These features improve the time efficiency of a rapid survey for large areas, and can reconstruct the underground three-dimensional images easily and quickly. The system is equipped with eight pairs of bowtie antennas. The antenna polarization is parallel to the direction of the survey line, and the antenna configuration is shown in Fig. 2. The rest parameters of the system are summarized in Table I. The theoretical spatial resolution of the multi-static GPR system in the air is $10 \mathrm{~cm}$. Since the system operates with the ultra-wideband bandwidth, not all the frequencies have the equivalent energy emitting out of the antenna. The actual spatial resolution of the system is approximately $15 \mathrm{~cm}$ in the air. When the system operates with a dielectric constant equal to 9 subsurface media, then the corresponding spatial resolution is $5 \mathrm{~cm}$.

\section{B. CMP Analysis and Survey Design}

The GPR data are usually obtained by either the variable offset or the common offset surveys. The variable offset measurements can be the common midpoint (CMP) or the 


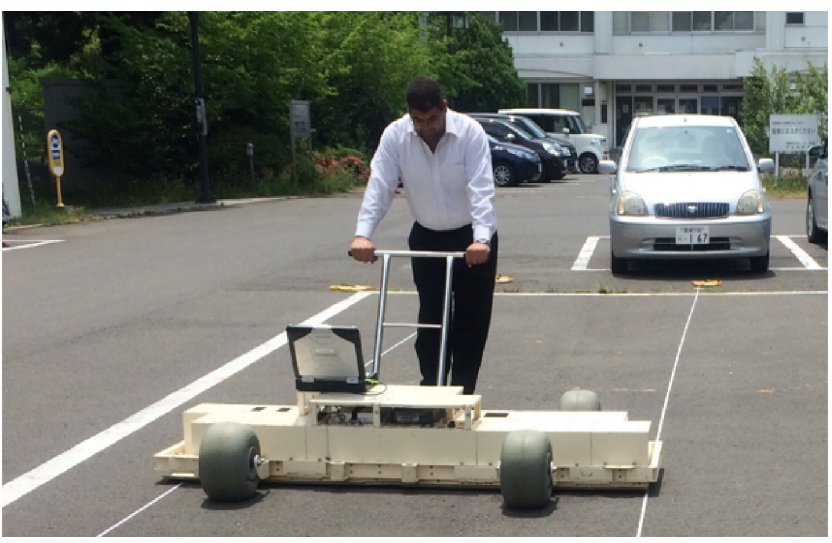

Fig. 1. The multi-static GPR system.

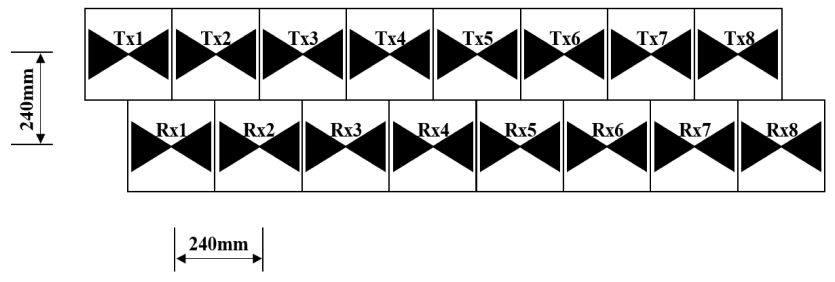

Fig. 2. The configuration of the 8 transmitting and 8 receiving antennas.

wide-angle reflection and refraction (WARR) measurements. First, the transmitting and receiving antennas are initially separated at a relatively small distance. When two antennas are gradually separated, CMP surveys are obtained by the additional measurements. For the WARR measurements, the transmitter and receiver also make the first measurements at a small distance, then one antenna remains stationary while the other antenna gradually moves away for the additional measurements.

The characteristics of the multi-static GPR system and the configuration of the antenna expand the possibility of the variable offset measurement. Each pair of antennas can simultaneously provide the traditional B-scan images along the multiple measuring lines. The multi-static data can be used directly in three-dimensional imaging. In addition, CMP gathers can be extracted and analyzed further. When the transmitting and receiving antennas are initially separated by the relatively small distance, CMP gathers are obtained, and then the two

TABLE I

Multi-STATIC GPR System PaRAMETERS

\begin{tabular}{cc}
\hline \hline Parameter & Value \\
\hline Frequency Range & $50 \mathrm{MHz}-1.5 \mathrm{GHz}$ \\
Penetration Depth & $2 \mathrm{~m}$ \\
Sampling Points & 256 \\
Sweeping Time & About $0.1 \mathrm{~s}$ \\
Survey Interval & $1 \mathrm{~cm} / \mathrm{trace}$ \\
Maximum Speed & $7 \mathrm{~km} / \mathrm{h}$ \\
System Type & Step Frequency Continues Wave (SFCW) \\
Antenna Type & Bowtie Antenna \\
Spatial Resolution & $10 \mathrm{~cm}$ (Air) \\
\hline \hline
\end{tabular}

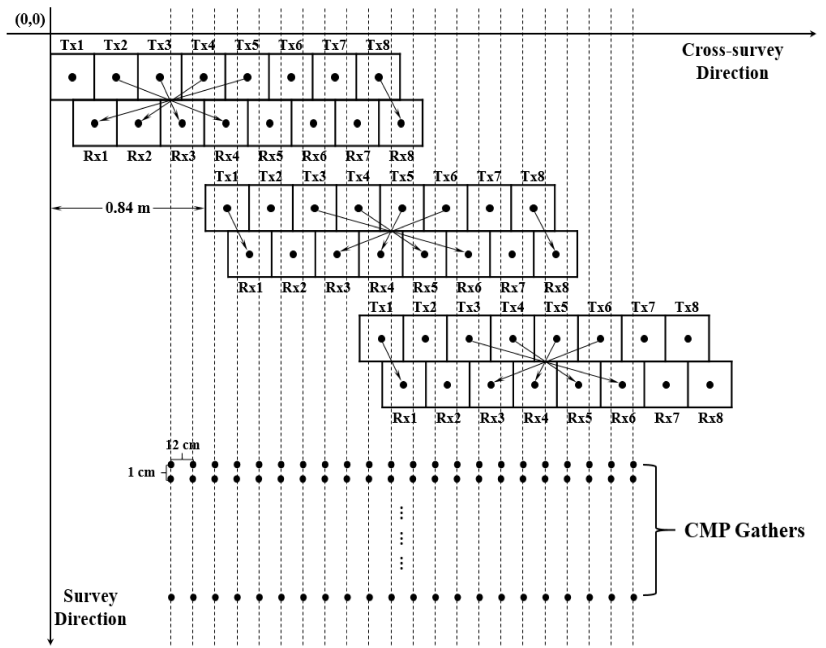

Fig. 3. The coordinate of the multi-static GPR measurement for the CMP gathers extraction.

antennas move away gradually when additional measurements are obtained. By considering the antenna configuration of the multi-static ground penetrating radar system and designing the distance between the measurement lines, CMP gathers can be obtained at each grid point of a dense grid. As shown in Fig. 3, three multi-static GPR surveys with the spacing of $84 \mathrm{~cm}$ are set. The Tx8-Rx8 pair reflection of the first survey matches with the 8 middle refection pair of the second survey. In that analogy, one CMP gather can be extracted from each of the measurement points in the dashed line in Fig.3. Therefore, dense CMP gathers can be obtained at a distance of $1 \mathrm{~cm}$ in the measurement direction and at a distance of $12 \mathrm{~cm}$ in the cross-measurement direction of the measurement area. For example, at the experimental site of Tokyo International Airport, about 18,000 CMP gatherings can be extracted in a survey area of 3 meters by 6 centimeters. In this case, the high-density CMP gathers can be extracted for further analysis through two-dimensional GPR measurements.

\section{Airport Asphalt Pavement Structures}

The construction of the airport pavement is to provide sufficient support for the loads imposed by the aircraft and to produce a solid, stable, smooth, year-round, all-weather surface, which can not be picked up by the jet explosion or the propeller cleaning. In order to meet these requirements, the pavement must have sufficient quality and thickness so that it will not fail under the applied loads. In addition, it must have sufficient inherent stability without damage to withstand the impact of traffic erosion, the bad weather conditions, and other deteriorations. To produce those pavements, many factors such as design, construction, and inspection, need to be coordinated to ensure the best combination of the available materials and high standards of the artistry [1].

Most airport pavements include a surface asphalt layer, a base asphalt layer, a stable granular "subbase" and a bottom "subgrade" layer. For some pavements, the base can also be composed of the bonding materials, but usually, only the 


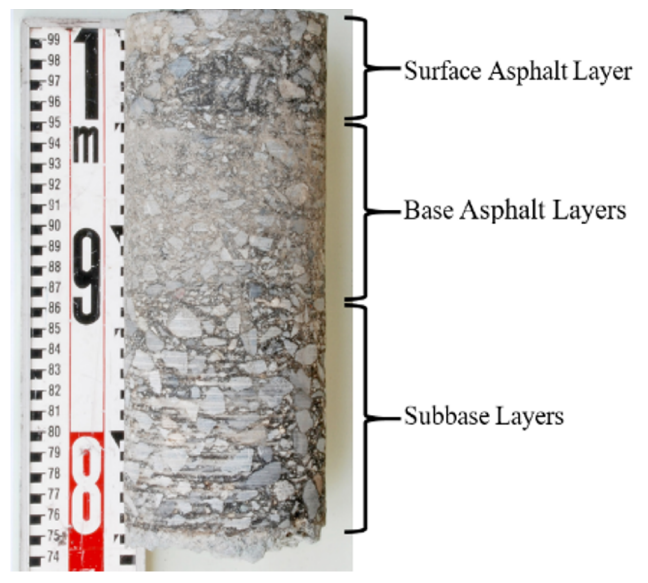

Fig. 4. A core sample showing layers structure at Tokyo International Airport taxiway asphalt pavement.

upper pavement structure is composed of the asphalt bonding materials or cement bonding materials. Figure 4 shows a core sample of the taxiway asphalt pavement at the Tokyo International Airport in Japan. In that sample, the bound and upper layer consists of two asphalt layers with $5 \mathrm{~cm}$ and $10 \mathrm{~cm}$ thickness, respectively. These asphalt layers provide the main structural strength and load propagation capability, which can reduce the stress applied by the aircraft to a level that can be maintained by the roadbed. The subbase bonding layer can be regarded as a single asphalt layer with tens of centimeters. Usually, most interlayer debonding occurs at the interface between the surface and the base asphalt layer, and a small part occurs at the interface between the base asphalt and the base.

\section{THEORY AND CONSIDERATIONS FOR LATERAL WAVE ANALYSIS}

In the above section, the structure of the multi-static GPR system and the airport asphalt pavement is described. At the same time, the method of extracting the dense CMP gathers from common measurements by the multi-static system is also introduced. In this section, we will discuss the theory, assumptions, and considerations of the lateral wave analysis by considering the specific application of the airport pavement inspection and the basic properties of the asphalt pavement.

\section{A. Lateral Wave}

GPR is a non-destructive detection technology, which uses the high-frequency electromagnetic energy to detect the underground non-invasively. The energy emitting from the GPR transmitter, a portion of them arrives at the receiver which records the energy received as a function of the time. When the transmitter is placed on the ground, the energy radiates a spherical wavefront through the air and the ground. The radiation rate of the wavefront depends on the dielectric constant of the medium. Because the electromagnetic field at the interface must be continuous, the incident, reflected and refracted waves cannot satisfy the requirements of the boundary conditions unless a plane wave is introduced on the boundary surface. For an incident wave to be a spherical wave, the boundary condition can only be satisfied if the electromagnetic surface wave, commonly known as the lateral wave, exists. Similarly, the evanescent wave generated by the soil wavefronts in the air should also be included. In fact, the main component of the electromagnetic field propagating along the boundary is the lateral wave [29]. In Fig. 5, the lateral wave can be considered as the tangent wavefront of the connection of the ground wave and the air wave at the interface, where both the air wave and the ground wave have the spherical wavefronts.

The one-way propagation of the lateral wave follows the behavior of $\frac{1}{\rho^{4}}$ ( $\rho$ : the distance to the source) and its loss is independent of the underground material. This is obviously a greater loss than the air and ground waves. However, the amplitude of the lateral wave near the surface will eventually exceed the amplitude of the ground wave after a large distance propagation. Based on this kind of property, the lateral wave has a special meaning to the forward-looking ground penetrating radar, and it is also important for reducing the surface clutter in the GPR survey data [25]. The propagation path of the lateral wave can be considered as the distance between the transmitting antenna and the receiving antenna [30]. The propagation velocity of the lateral wave can be used to estimate the electromagnetic properties of the near-surface materials. Based on the appropriate approximation under low loss conditions, the lateral wave velocity has been used to estimate the dielectric constant [31].

The method of the variable offset measurement is also very important for interpreting the lateral radar waves. They allow the properties of the subsurface material to be directly estimated by the properties of the lateral wave as the antenna offset changes. In addition, the lateral wave propagates tens of centimeters below the subsurface material in the GPR operating bandwidth. These features together make the lateral wave playing an important aspect for the analysis of the properties of the shallow subsurface regions. Some researchers have used the variable offset data to measure the lateral wave velocity, which is then used to estimate the soil water content [32][33]. In this paper, by considering the variation of the lateral wave energy with the antenna offset, a direct method for detecting small anomaly occurrences in the shallow pavement structure by a multi-static GPR system is given. Since the lateral wave is generated only at the boundary of the twomaterial boundary, it is very sensitive to the performance of the interface structure and the material properties. Before carrying out the specific analysis, it is necessary to avoid some interference and perform an appropriate calibration. In the following subsections, the consideration and the calibration procedures will be discussed and given based on the specific application of the airport pavement inspection.

\section{B. Air Wave}

In the GPR record, the energy emits from the transmitter, propagates through the material interface, generates different waves and propagates through different paths to the receiver. The air wave travels in a straight path between the transmitter 


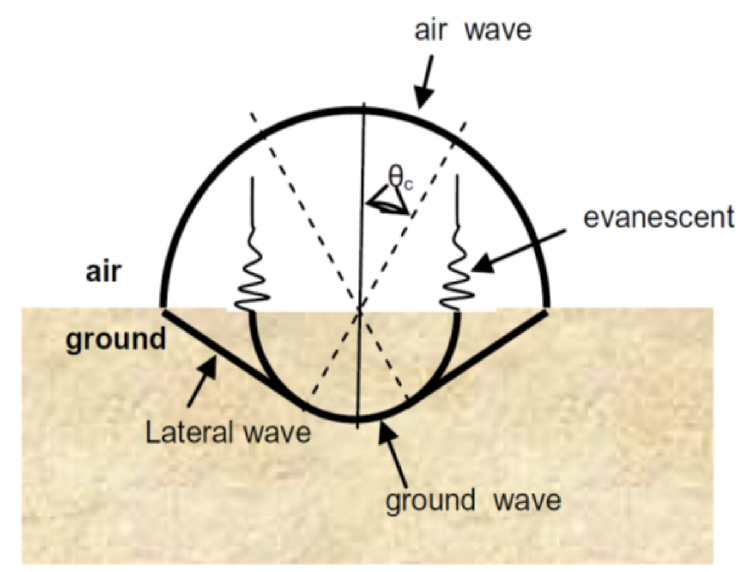

Fig. 5. Physical interpretation: Air wave, Ground wave, Lateral wave, and Evanescent wave [25][29].

and the receiver with the electromagnetic wave in a vacuum $\left(3 \times 10^{8} \mathrm{~m} / \mathrm{s}\right)$. Due to the fastest propagation speed, it is the first energy recorded by the GPR receiver. The lateral wave propagates along with the near-surface medium. Because of its short propagation path, it arrives at the receiver after the air wave. When a contrast in the electromagnetic properties exits in the subsurface, the reflected and refracted waves are also generated.

Based on the antenna configuration and the working bandwidth of the multi-static GPR system, the air waves are always mixed with the lateral waves. In order to extract the lateral wave, the air wave must be subtracted from the original GPR signal. We assume that the material of the pavement is frequency-independent at the operating frequency of the multistatic GPR system and the coupling factors of the transmitting and receiving antennas are the same. However, for different measurement conditions, such as the surface roughness and the antenna off-plane distance, the radiation pattern is slightly different [34][35]. Based on the above assumptions, the air wave acquired for different conditions can be equal to a ratio of the air wave which is acquired at the free space. In this paper, the free-space measurement is conducted to obtain the airwave, and the obtained air wave is then subtracted from the original GPR record.

\section{Consideration and Calibration}

In the ideal case, the amplitude curve of the processed GPR data is directly represented by the reflection coefficient. However, there are some unrelated factors that will affect the amplitude recorded at the target boundary. In general, the amplitude information recorded by the GPR can be expressed as $A m p_{\text {obs }}$ [36]

$$
A m p_{\text {obs }}=\frac{A_{T} \cdot A_{R} \cdot C_{T} \cdot C_{R} \cdot T}{G} \cdot r \cdot e^{-\alpha \cdot \rho} \cdot A m p_{\text {sou }} \text {. }
$$

Here $A_{T}$ and $A_{R}$ are the radiation pattern of the transmitter and the receiver antennas, respectively. $T$ is the transmission loss across the boundaries in the overlay. $G$ is the geometric spreading factor (also called Green Function) and $r$ is the reflection coefficient. $A m p_{\text {sou }}$ denotes the amplitude of the source signal. $\alpha$ is the attenuation coefficient. $\rho$ is the length of the propagation path. $C_{T}$ and $C_{R}$ are the coupling factor of the transmitter and the receiver antennas, which are the measure of the energy loss at the antenna/earth interface.

Each parameter in (1) can be a function of the frequency, dielectric constant, conductivity, magnetic permeability, polarization, and the ray parameters. In addition, the coupling factor is a function of the surface roughness. The dielectric constant, conductivity and magnetic permeability are functions of the frequency. Obviously, the amplitude data observed by GPR is an extremely complex function of many variables, and most of them are difficult or impossible to determine. However, the potential task of this study was to detect the interlayer debonding in the airport asphalt pavement in advance. By considering the nature of the airport asphalt pavement and the purpose of the investigation, we can reduce the complexity of the function greatly.

First of all, assume that the coupling factor does not change significantly with the offset, the coupling factor and the radiation pattern of all the transmit and receive antennas are the same. Since the surface of the airport asphalt pavement is flat and the material is horizontally uniform, that assumption is also established in the airport measurement.

Next, we assume that the transmission loss does not change significantly with the offset, which means that the strata in the overlay are flat-lying and lateral uniform. The transmission loss includes not only the attenuation inside the asphalt but also the intrinsic attenuation of the near-surface. Therefore, it requires the smoothness of the surface of the pavement to be equivalent to one wavelet. On the other hand, from a purely theoretical point of view, the attenuation coefficient $\alpha$ can be expressed by the dielectric constant $\varepsilon$, and the permeability $\mu$ of the asphalt. The $\alpha$ of the airport asphalt pavement can be considered as a constant [37]

$$
\alpha \simeq 0.31=\text { constant }
$$

Last, assume that the asphalt is a frequency-independent material in the system's operating bandwidth. The frequency independence is a significant assumption and also a reasonable assumption for the airport asphalt pavement. In [37], the relative permittivity $\varepsilon$ of the airport asphalt pavement ranging from $30 \mathrm{MHz}$ to $20 \mathrm{GHz}$ is about

$$
\varepsilon \simeq 4=\text { constant }
$$

Actually, the imaginary part associated with the attenuation coefficient $\varepsilon$ can also be assumed to be a constant value in the operating bandwidth of the system [38].

Based on the above assumptions, many factors affecting the lateral wave amplitude and the reflection coefficient can be eliminated when evaluating the variation of the relative amplitude of the lateral wave along with the offset. This allows us to calculate the radiation pattern, the geometric spreading function and the attenuation for the next correction.

The correction of the antenna radiation pattern is especially important in this study. In general, the antenna radiation pattern correction requires the numerical simulating the antenna under the specific condition. Attenuation correction still needs 
careful consideration. It is very difficult to separate the reflectivity, transmission loss, antenna coupling and the near-surface effects from the intrinsic attenuation. Ideally, we calculate the attenuation coefficient from the conductivity measurements in the laboratory or the field. However, those measurements are usually not easy to be carried out. If the frequency-independent attenuation assumption is approximately correct over the GPR working bandwidth, a simple exponential gain correction can be applied. Some researchers also pointed out that ignoring the frequency-dependent attenuation coefficient is reasonable in the GPR working bandwidth [36]. Even in the case when the frequency dependence cannot be ignored, the attenuation can be approximated as a linear function of the frequency variation of the bandwidth of the GPR signal.

In general, it is impossible to find an absolute solution for the amplitude correction in the practical applications. In this article, we implemented a solution for the specific purpose application, which is the core of this paper. The background response of the GPR signal can be acquired at the sounding asphalt pavement

$$
A m p_{b g}=\frac{A_{T} \cdot A_{R} \cdot C_{T} \cdot C_{R} \cdot T}{G_{b g}} \cdot r \cdot e^{-\alpha \cdot \rho} \cdot A m p_{\text {sou }}
$$

where $G_{b g}$ is the geometric spreading factor of the background.

For the lateral wave response of the airport asphalt pavement acquired at any fix offset, all the related parameters could be assumed equal except the geometric spreading factor or the Green function. Therefore, taking the ratio of the later wave curve to the background curve response for the amplitude correction procedure, we could get

$$
\frac{\text { Amplateral }}{A m p_{b g}} \simeq \frac{G_{b g}}{G_{\text {lateral }}}+\text { noise. }
$$

Moreover, taking the ratio of the target curve to the background curve response to cancel the effect of the antenna radiation pattern is much more suitable for the real application. The proposed approach needs the multi-static GPR system to acquire the CMP gathers as much as possible inside the survey site and then identify the reflectivity anomalies by the details investigation with the limited local CMP gathers. The method is the most effective with continuous CMP profiling throughout the survey area. It allows us to combine the adjacent CMP gathers to improve the $\mathrm{S} / \mathrm{N}$ ratio. Our method enables the laterally continuous evaluation of the offset depending on the energy attributes and provides a statistical basis for the error estimates.

Other processing artifacts should also be considered when measuring the field-data amplitude. Typically, when the little interference occurs over a large incidence angle, the lowfrequency evanescent wave has a significant effect on the offset-dependent amplitude curve of the shallow region. These potential processing or noise artifacts should be considered carefully on a basic case by case. In a practical view, although the system could provide 8 traces CMP gathers up to $1.8 \mathrm{~m}$ offset, only 3 to 4 near traces CMP gather should be used to detect the anomaly.

\section{RESUlTS AND Discussion}

To validate the idea proposed in Section III, both the simulation and the field measurement are conducted. Moreover, we compare the quality of the detection results obtained by the proposed approach with the conventional acoustic finding method. In the last, the validation by the coring on site is described.

\section{A. Numerical Simulation}

Based on the airport asphalt pavement structures described in Section II, the sound asphalt pavement can be assumed as a three-layered medium that consists of the air, the surface layer, and the based layer. While the interlayer debonding asphalt pavement could be assumed as a four-layered medium that consists of the air, the surface layer, the thin air/water layer, and the based layer. Although the mathematical formulation of the lateral wave propagates along the layered medium are given by researchers [39]-[42], existing the ultra-thin layer (less than $1 \mathrm{~mm}$ ) make it difficult to obtain a correct solution in the GPR working bandwidth.

In the simulation, a three-layers model (Fig. 6(a)) and a four-layers model (Fig. 6(b)) were given to simulate the sound and interlayer debonding occurred pavement in the airport, respectively. In the four layers model, a $1 \mathrm{~mm}$ thickness gap was added between the surface and the based asphalt layer. Both the air and the water gap models were simulated. A half wavelength horizontal dipole with the frequency $500 \mathrm{MHz}$ was placed $1 \mathrm{~mm}$ above the surface. The simulation was carried out under the CST Studio Suite which is a high-performance 3D EM analysis software package for designing, analyzing and optimizing the electromagnetic (EM) components and systems. $500 \mathrm{MHz}$ is corresponding to the central frequency of the multi-static GPR system. The simulation was carried out in the frequency domain with a frequency-domain solver of the software. The simulation parameters are summarized in TABLE II.

To consist of the multi-static GPR system, the electric field density besides the half-wavelength dipole $12 \mathrm{~cm}$ away was recorded. Fig. 7(a) shows the top view of the simulation model. The dot points indicate the electric field density monitoring points. The air direct wave was subtracted with the theoretical value. Fig. 7(b) shows the electric field density varies with the distance of different models. In Fig. 7(b), the dashed line with a plus sign indicates the three layers model responses, the dashed line with a circle sign indicates the four layers model with the air gap responses and the dashed line with a square sign indicates the four layers model with the water gap responses. Compare with the three layers model (the sound asphalt pavement) responses, the four layers model (the interlayer debonding asphalt pavement) responses have a various tendency with the distance. These differences can be used for the interlayer debonding detection of the pavement. In the following, the various rate of the lateral wave with the offset will be used for the detection, and the detail will be described by the field data acquired at Tokyo International Airport. 


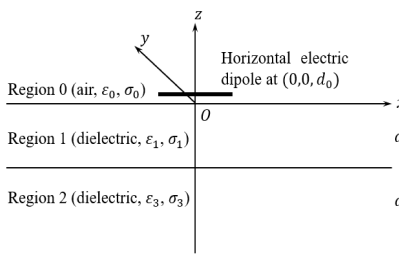

(a) (b)

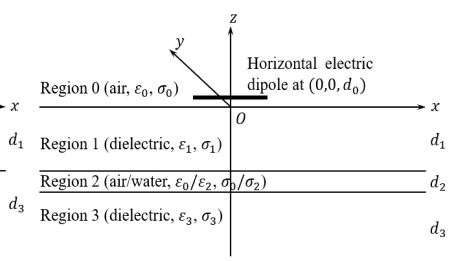

Fig. 6. The simulated layered models; (a) three layered model represents the sound airport pavement structure; (b) four layered model represents the defect airport pavement structure.

TABLE II

PARAMETERS OF THE MODELS FOR SiMULATION

\begin{tabular}{ccc}
\hline \hline Parameter & Three Layers Model & Four Layers Model \\
\hline$\varepsilon_{0}$ & 1 & 1 \\
$\varepsilon_{1}$ & 9 & 9 \\
$\varepsilon_{2}$ & $/$ & 1 or 81 \\
$\varepsilon_{3}$ & 16 & 16 \\
$\sigma_{0}$ & 0 & 0 \\
$\sigma_{1}$ & $10^{-8} \mathrm{~s} / \mathrm{m}$ & $10^{-8} \mathrm{~s} / \mathrm{m}$ \\
$\sigma_{2}$ & $/$ & 0 or $0.01 \mathrm{~s} / \mathrm{m}$ \\
$\sigma_{3}$ & $10^{-8} \mathrm{~s} / \mathrm{m}$ & $10^{-8} \mathrm{~s} / \mathrm{m}$ \\
$d_{0}$ & $0.1 \mathrm{~cm}$ & $0.1 \mathrm{~cm}$ \\
$d_{1}$ & $5 \mathrm{~cm}$ & $5 \mathrm{~cm}$ \\
$d_{2}$ & $/$ & $0.1 \mathrm{~cm}$ \\
$d_{3}$ & $25 \mathrm{~cm}$ & $25 \mathrm{~cm}$ \\
\hline \hline
\end{tabular}

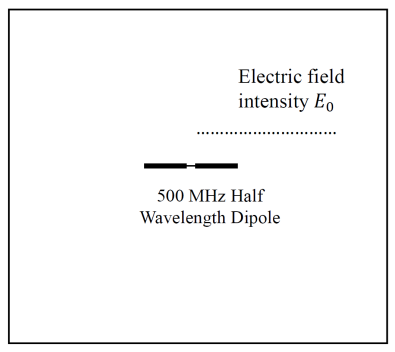

(a)

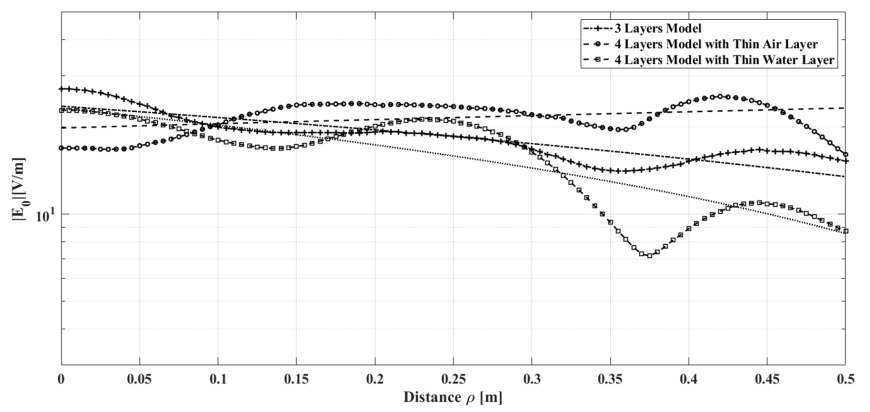

(b)

Fig. 7. The simulated layered models; (a) three layered model represents the sound airport pavement structure; (b) four layered model represents the defect airport pavement structure.

\section{B. Field Measurement}

The measurement was carried out on the taxiway asphalt pavement at Tokyo International airport to test the feasibility of the proposed approach. The measurement was carried at midnight and it was shown in Fig. 8(a). The size of the entire measurement area was around $20 \mathrm{~m}^{2}$ which was shown in Fig. 8(b). The data consisted of three parallel multi-static GPR acquisitions along the direction of the aircraft moving. We chose the survey area that contained the known sound pavement and the interlayer debonding pavement parts. The interlayer debonding occurring areas located at $4 \mathrm{~m}$ to $6 \mathrm{~m}$ in the $\mathrm{x}$-direction (survey direction) and $4.5 \mathrm{~m}$ to $5.5 \mathrm{~m}$ in the $y$-direction (the cross-survey direction), was shown as the white circles in Fig. 8(a).

Figure 9 shows a GPR profile acquired at $5.08 \mathrm{~m}$ in the $\mathrm{x}$ direction. In Fig. 8(a), we can see that $5.08 \mathrm{~m}$ is the line across the interlayer debonding part. It is very difficult to observe the anomaly through this profile. As mentioned in [43], the main difficulty with the data processing relies on the reflection detection of the signal backscattered to detect from the interlayer debonding.

To eliminate the potential for the amplitude artifacts, we extract the amplitudes from the peak of the envelop signal. The amplitude we use for the offset changing analysis is the maximum local value of the envelope function within a time gate bounding the lateral wave, 0 to 5 ns shown in Fig. 10 . After the air coupling substation and the amplitude correction mentioned in Section III, the parameters of the linear function are estimated by the least square method

$$
\min _{a}\left\|a \cdot \rho-A m p_{\max }\right\|_{2}=0
$$

where $\rho$ indicates the antenna offset distance and $A m p_{\max }$ is the corresponding maximum amplitude of the lateral wave. While $a$ is the main variable we concerned. Normally, $a$ is a constant value around 0 which indicates that no thin layer is embed in the subsurface, shown in Fig. 10(a). Once $a$ shows a large positive or negative value, it means the received lateral wave energy is increasing or decreasing with the offset. It is a typical phenomenon of the thin layer embedded in the subsurface, shown in Fig. 10(b).

The gradient value $a$ of the maximum value of the lateral wave changing along the offset of the whole measurement area is shown in Fig. 11(a). In Fig. 11(a), the higher amplitude appears in the y-direction of around $3 \mathrm{~m}$ to $5 \mathrm{~m}$ and in the x-direction between $4.5 \mathrm{~m}$ and $5.5 \mathrm{~m}$, which makes us believe that the interlayer debonding occurred. Figure 11(b) shows the five possible debonding points which are detected by the conventional acoustic sounding method. These five-points locations match well with the high-value zones in Fig. 11(a). In the middle of Fig. 11(a), a large zone shows high value of $a$. But, it was not detected by the conventional acoustic sounding method. We expect that in this area, the earliest stage of interlayer debonding has occurred. While this earliest stage defect in the asphalt pavement cannot be detected by the conventional acoustic sounding method, but the proposed approach can detect it.

\section{Validation by Coring}

At Tokyo International Airport, the inspection by the conventional acoustic finding method has been commonly used, and several operators hit the surface of the pavement by the 


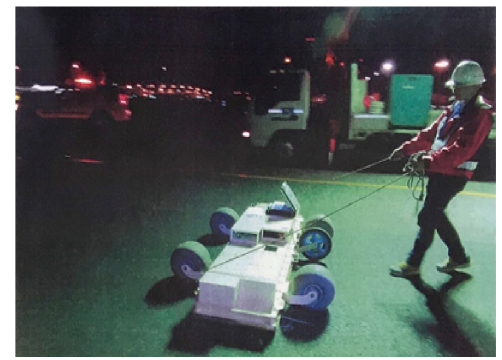

(a)

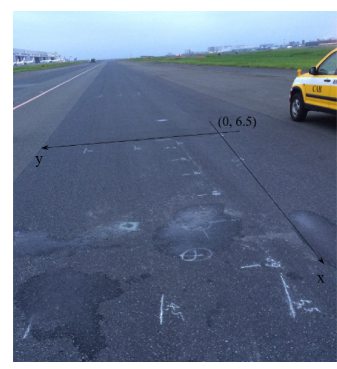

(b)
Fig. 8. Field measurement at Tokyo International Airport; (a) the operation of the multi-static GPR system in site; (b) the whole measurement site. The white circles indicate the anomaly detected by the conventional acoustic finding method.

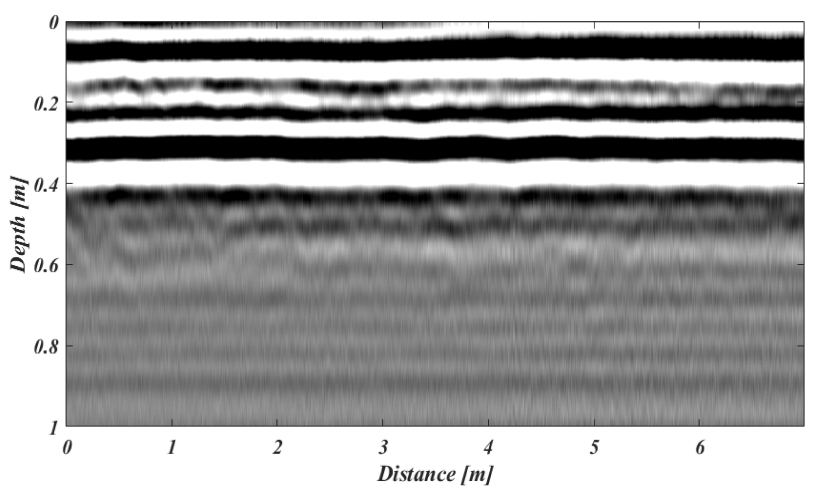

Fig. 9. The GPR profile acquired at $5.08 \mathrm{~m}$ in the $\mathrm{x}$-direction (crossing the interlayer debonding part).

metal rods hearing the anomalous sound. It spends about three weeks to complete the inspection of all the pavements.

The five points are shown as the white circles in Fig. 8(a) and Fig. 11(b) are pointed out by the conventional acoustic finding method. Two of them located in the area around $5 \mathrm{~m}$ in the $\mathrm{x}$-direction and $5 \mathrm{~m}$ in $\mathrm{y}$-direction selected to the core. Fig. 12(a) and (b) present the two cores which are cored by a drilling machine during the night. From the cylindrical cores, we could see that the damaged parts mainly occur at $5 \mathrm{~cm}$ and $20 \mathrm{~cm}$ in depth, as shown in Fig. 12(c) and (d). Meanwhile, the damaged crack can be seen from the sidewall coring shown as the white line in Fig. 12(e) and (f). The coring area matches with the anomaly parts that we point through the detection results by the proposed approach.

\section{CONCLUSION}

In this paper, we develop a new method to evaluate the defects or anomalies occurred inside the pavement. The method characterizes the properties of the lateral wave in the CMP gathers acquired by a multi-static GPR system. The multistatic GPR system and the strategy for the dense CMP gather acquisition with the time-efficient, and the high-resolution characteristic is introduced. With the developing signal processing method, we analyze the behavior of the lateral wave of the CMP gathers to detect the interlayer debonding inside the taxiway pavement. Since the lateral wave is very sensitive to the material property, the assumption, the consideration, and the calibration procedures are also discussed by considering

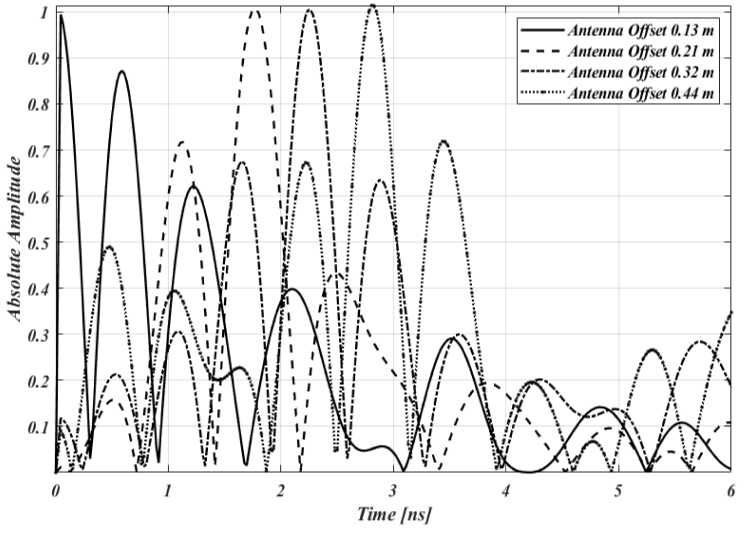

(a)

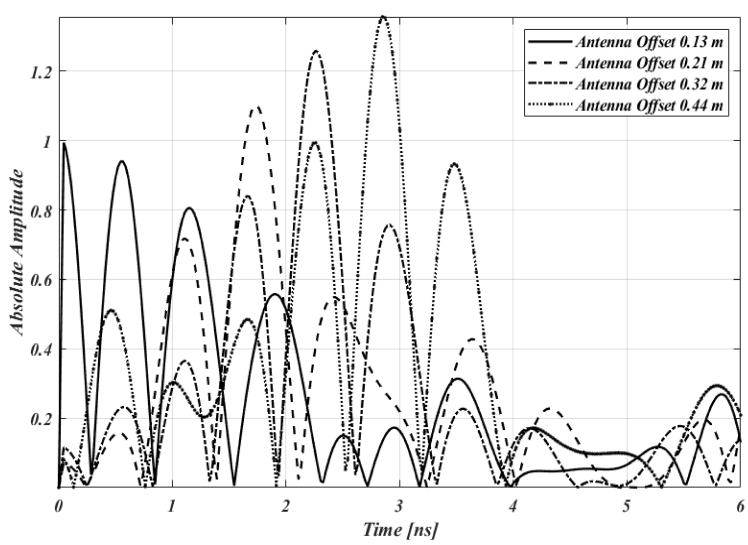

(b)

Fig. 10. The lateral wave of CMP gather after the amplitude correction; (a) the lateral waves acquired at the sound area; (b) the lateral waves acquired at the interlayer debonding area.

the asphalt pavement properties and the specific application of the airport pavement inspection. Moreover, the variation of the maximum amplitude value is estimated as a linear function of the antenna offset by the least square method. Finally, the gradient variation map of the maximum amplitude various with the antenna offset is obtained. With the real data acquired at the airport taxiway pavement, the proposed approach shows a good performance for detecting the interlayer debonding of the upper asphalt layers. Furthermore, the results have a highlevel consistent with the conventional acoustic finding method. The coring on the site also shows the excellent performance of the proposed approach. Compared to the conventional acoustic finding method, the approach proposed with the multi-static GPR system in this paper can finish a square with $10 \mathrm{~m}$ by 10 $\mathrm{m}$ in several minutes. It is a much more efficient approach for the nondestructive inspection in the airport asphalt pavement.

\section{REFERENCES}

[1] G. Weil, "Nondestructive testing of airport concrete structures (runways, taxiways, roads, bridges \& building walls and roofs)," in Proc. of the 7th International Conference on Ground Penetrating Radar (GPR'98), Lawrence, USA, May 1998, pp. 443-448.

[2] L. Yi, L. Zou, and M. Sato, "A simplified velocity estimation method for monitoring the damaged pavement by a multistatic GPR system YAKUMO," in Proc. of the 17th International Conference on Ground Penetrating Radar (GPR2018), Rapperswil, Switzerland, Jun. 2018, pp. $1-4$. 


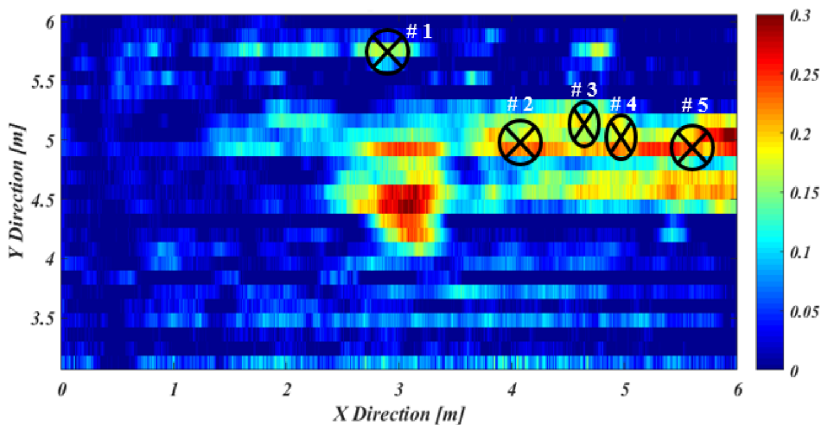

(a)

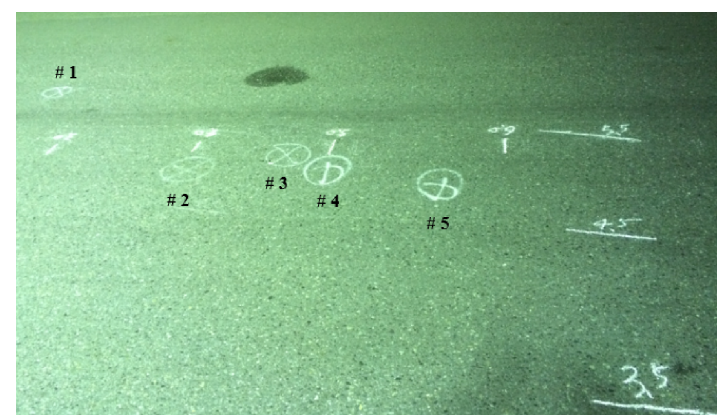

(b)

Fig. 11. Interlayer debonding detection results; (a) the detected result by the proposed approach; (b) the detected result by the conventional acoustic finding method.

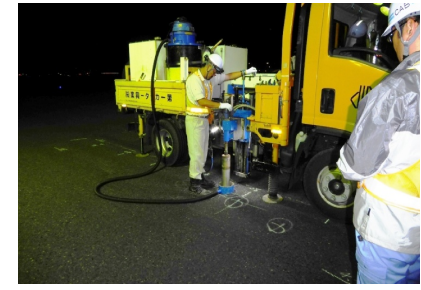

(a)

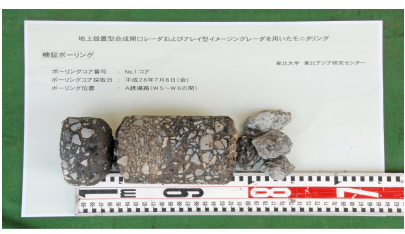

(c)

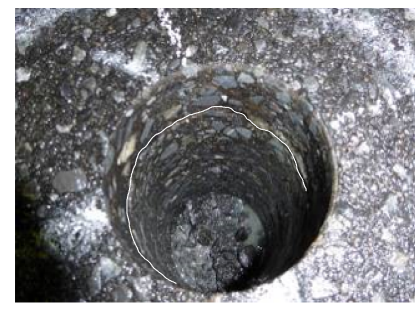

(e)

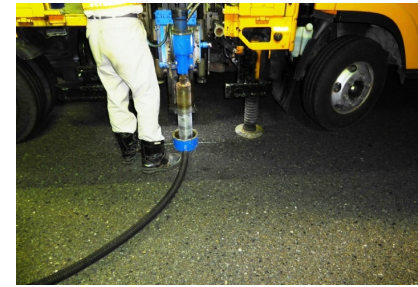

(b)

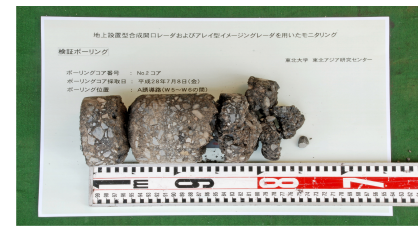

(d)

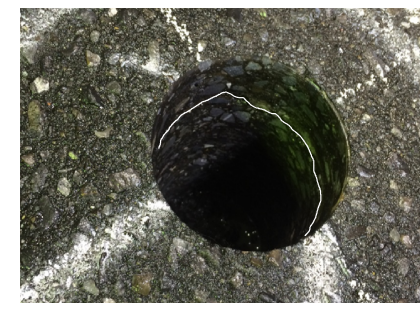

(f)
Fig. 12. Coring on the taxiway asphalt pavement.

[3] L. Zou, K. Kikuta, and M. Sato, "Nondestructive Inspection of an Airport Pavement by MIMO Array GPR YAKUMO," in Proc. of the 17th International Conference on Ground Penetrating Radar (GPR2018), Rapperswil, Switzerland, Jun. 2018, pp. 1-4.

[4] E. Schnebele, B. F. Tanyu, G. Cervone, and N. Waters, "Review of re- mote sensing methodologies for pavement management and assessment,' Eur. Transp. Res. Rev., vol. 7, no. 2, pp. 1-19, Mar. 2015.

[5] D. Breysse, "Nondestructive evaluation of concrete strength: An historical review and a new perspective by combining NDT methods," Construction Building Mater, vol. 33, pp. 139-163, Aug. 2012.

[6] H. M. Jol, Ground penetrating radar theory and applications. Amsterdam, The Netherlands: Elsevier, 2008.

[7] D. Daniels, Ground Penetrating Radar, 2nd ed. London, U.K.: The Inst. Elect. Eng. (IEE), 2004.

[8] R. Evans, M. Frost, M. Stonecliffe-Jones, and N. Dixon, "A review of pavement assessment using ground penetrating radar (GPR)," in Proc. of the 12th International Conference on Ground Penetrating Radar (GPR2008), Birmingham, UK, Jun. 2008, pp. 1-10.

[9] C. Plati, K. Georgouli, and A. Loizos, "Review of NDT assessment of road pavements using GPR," Nondestruct. Testing Mater. Struct., vol. 6, pp. 855-860, Sep. 2013.

[10] A. Szynkiewicz and P. Grabowski, "GPR monitoring of pavements on airfield," in Proc. of the 10th International Conference on Ground Penetrating Radar (GPR2008), Delft, The Netherlands, Jun. 2004, pp. 21-24.

[11] T. Saarenketo and T. Scullion, "Road evaluation with ground penetrating radar," J. Appl. Geophys., vol. 43, no. 2, pp. 119-138, Mar. 2000.

[12] Z. Deng and M. Sato, "GPR reflection signal from a thin layer," in Proc. of the SEG-J Conference 134th, Tokyo, Japan, May 2016, pp. 47-50.

[13] H. Liu, Z. Deng, F. Han, Y. Xia, Q. Liu, and M. Sato, "Time-frequency analysis of air-coupled GPR data for identification of delamination between pavement layers," Construction Building Mater, vol. 154, pp. 1207-1215, Nov. 2017.

[14] H. Liu and M. Sato, "In situ measurement of pavement thickness and dielectric permittivity by GPR using an antenna array," NDT\&E Int. vol. 64, pp. 65-71, Jun. 2014.

[15] S. Sanders, D. Rath, and F. Parker, "Comparison of nuclear and core pavement density measurement," J. Transp. Eng., vol. 120, no. 6, pp. 953-966, Nov. 1994.

[16] R. Sztukiewicz, "Application of ultrasonic methods in asphalt concrete testing," Ultrasonics, vol. 29, no. 1, pp. 5-12, Jan. 1991.

[17] T. Jiang, Q. Kong, D. Patil, Z. Luo, L. Huo, and G. Song, "Detection of debonding between fiber reinforced polymer bar and concrete structure using piezoceramic transducers and wavelet packet analysis," IEEE Sensors J., vol. 17, no. 7, pp. 1992-8, Jan. 2017.

[18] S. Hiasa, R. Birgul, and F. Catbas, "Infrared thermography for civil structural assessment: demonstrations with laboratory and field studies," J. Civ. Struct. Health Monit., vol. 6, no. 3, pp. 619-636, Jul. 2016.

[19] H. V. Quintus, NDT technology for quality assurance of HMA pavement construction. Transportation Research Boards, 2009.

[20] A. Loizos and C. Plati, "Accuracy of pavement thicknesses estimation using different Ground Penetrating Radar analysis approaches," NDT\& E Int., vol. 40, no. 2, pp. 147-157, Mar. 2007.

[21] B. Beaucamp, C. Fauchard, and L. Laguerre, "Non destructive assessment of hot mix asphalt compaction/density with a step-frequency radar: Case study on a newly-paved road," Near Surf. Geophys., vol. 13, no. 3, pp. 289-297, Jun. 2015.

[22] C. Plati and A. Loizos, "Estimation of in-situ density and moisture content in HMA pavements based on GPR trace reflection amplitude using different frequencies," J. Appl. Geophys., vol. 97, pp. 3-10, Oct. 2013.

[23] T. Yu and O. B, "A far-field airborne radar NDT technique for detecting debonding in GFRP-retrofitted concrete structures," NDT\&E Int., vol. 41, no. 1, pp. 10-24, Jan. 2008.

[24] L. Yi, L. Zou, and M. Sato, "Practical approach for high-resolution airport pavement inspection with the yakumo multistatic array groundpenetrating radar system," Sensors, vol. 18, no. 8, p. 2684, Aug. 2018.

[25] C. C. Chen, "Lateral Waves in Ground Penetrating Radar Applications," in Proc. of the 14th International Conference on Ground Penetrating Radar (GPR2012), Shanghai, China, Jun. 2012, pp. 39-41.

[26] K. Grote, C. Taylor, and C. Nickel, "Experimental estimation of the GPR groundwave sampling depth," Water Resour. Res., vol. 46, no. 10, p. W10520, Oct. 2010.

[27] M. Sato, L. Yi, Y. Iitsuka, L. Zou, and K. Takahashi, "Optimization of antenna polarization of the multistatic GPR system "yakumo"," in Proc. of the 16th International Conference on Ground Penetrating Radar (GPR2016), Hong Kong, China, Jun. 2016, pp. 1-6.

[28] H. Liu, L. Zou, K. Takahashi, M. Sato, and J. Chen, "Development of an Array GPR System for Large-scale Archaeological Investigations," in Proc. of the 11th SEGJ International Symposium, Yokohama, Japan, Nov. 2013, pp. 1-4. 
[29] R. Shavit and E. Rosen, "Lateral Wave Contribution to the Radiation from a Dielectric Half Medium," IEEE Trans. on Antennas and Propagation, vol. 43, no. 7, pp. 751-755, Jul. 1995.

[30] A. P. Annan, "Radio interferometry depth sounding: Part I-Theoretical discussion," Geophysics, vol. 38, no. 3, pp. 557-580, Jun. 1973.

[31] C. Sperl, K. Stanjek, and A. Berktold, "Subsurface moisture determination with the ground wave of GPR," in Proc. of the 7th International Conference on Ground Penetrating Radar (GPR'98), Lawrence, USA, May 1998, pp. 675-680.

[32] H. Gerhards, U. Wollschlager, Q. Yu, P. Schiwek, X. Pan, and K. Roth, "Continuous and simultaneous measurement of reflector depth and average soil-water content with multichannel ground-penetrating radar," Geophysics, vol. 73, no. 4, pp. J15-J23, Jun. 2008.

[33] A. Klotzsche, F. Jonard, M. Looms, J. Kruk, and J. Huisman, "Measuring soil water content with ground penetrating radar: a decade of progress," Vadose Zone Journal, vol. 17, no. 1, Jun. 2018.

[34] D. Coster, Albric, and S. Lambot, "Full-Wave Removal of Internal Antenna Effects and Antenna-Medium Interactions for Improved GroundPenetrating Radar Imaging," IEEE Trans. Geosci. Remote Sensing, vol. 57, no. 1, pp. 93-103, Jul. 2019.

[35] S. J. Radzevicius, J. J. Daniels, and C. C. Chen, "Subsurface moisture determination with the ground wave of GPR," in Proc. of the 8th International Conference on Ground Penetrating Radar (GPR2000), Gold Coast, Australia, May 2000, pp. 712-717.

[36] J. H. Bradford and J. C. Deeds, "Ground-penetrating radar theory and application of thin-bed offset-dependent reflectivity," Geophysics, vol. 71, no. 3, pp. K47-K57, May 2006.

[37] T. Yamaguchi, T. Mizutani, M. Tarumi, and D. Su, "Sensitive Damage Detection of Reinforced Concrete Bridge Slab by Time-Variant Deconvolution of SHF-Band Radar Signal," IEEE Trans. Geosci. Remote Sensing, vol. 57, no. 3, pp. 1478-1488, Mar. 2019.

[38] E. J. Jaselskis, J. Grigas, and A. Brilingas, "Dielectric properties of asphalt pavement," J. Mater. Civil Eng., vol. 15, no. 5, pp. 2469-2480, Sep./Oct. 2003.

[39] T. T. Wu and R. W. P. King, "Lateral waves: a new formula and interference patterns," Radio Science, vol. 17, no. 3, pp. 521-531, MayJun. 1982.

[40] R. Shavit and E. Rosen, "Lateral Wave Contribution to the Radiation from a Dielectric Half Medium," IEEE Trans. on Antennas and Propagation, vol. 43, no. 7, pp. 751-755, Jul. 1995.

[41] J. M. Dunn, "Lateral waves propagation in a three-layered medium," Radio Science, vol. 21, no. 5, pp. 787-796, Sep.-Oct. 1986.

[42] K. Li, Electromagnetic Fields in Stratified Media. Hangzhou, China: Zhejiang University Press, 2009.

[43] R. Morey, Ground penetrating radar for evaluating subsurface conditions for transportation facilities. Transportation Research Board, 1998.

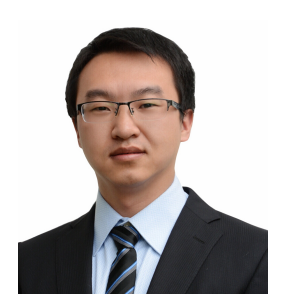

Lilong Zou (M'18) received the B.S. and M.S degrees from the College of Geo-Exploration Science and Technology, Jilin University, Changchun, China, in 2009 and 2012, respectively, and the Ph.D. degree in environmental studies from the Graduate School of Environmental Studies, Tohoku University, Sendai, Japan, in 2016.

From 2016 to 2018 , he was an assistant professor with Tohoku University, Japan. From 2018 to 2019 , he was a researcher with the National Institute of Advanced Industrial Science and Technology (AIST), Koriyama, Japan. He is currently a Research Fellow with School of Computing and Engineering, University of West London, London, U.K.. His research interests include signal processing technology of Ground Penetrating Radar (GPR), Interferometric and Polarimetric Ground-Based Synthetic Aperture Radar (GB-SAR) and their applications and analysis methods for disaster mitigation and nondestructive testing, and data processing technology of microseismic monitoring.

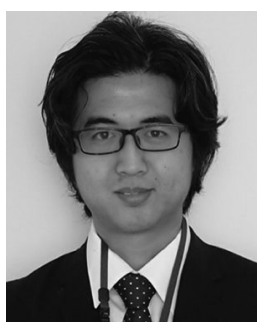

Li Yi (M'14) is an Assistant Professor of Information Photonics at Osaka university, Japan. He received the B.Sc. degree in geophysics from the School of Ocean and Earth Science, TongJi University, Shanghai, China, in 2011, and the M.E. and Ph.D degrees in environmental studies from the Graduate School of Environmental Studies, Tohoku University, Sendai, Japan, in 2014 and 2017, respectively. From 2017 to 2018 , he was a researcher with the National Institute of Advanced Industrial Science and Technology (AIST), Koriyama, Japan. Then he became an assistant professor with Osaka University, Japan.

His current research interests are imaging and signal processing methods with millimeter and terahertz waves, which include inverse problems, nondestructive inspection applications, and passive imaging approaches.

Dr. Yi received the student paper competition prize of URSI Japan Radio Science Meeting 2015 and was awarded the president award of environmental study of Tohoku University in 2017. And The young scientists award of IEICE, SANE in 2018.

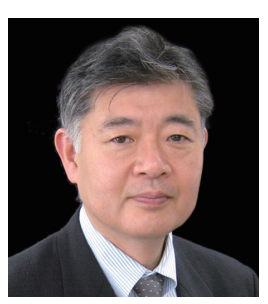

Motoyuki Sato (S'79-M'80-SM'02-F'10) received the B.E., M.E. degrees, and Dr. Eng. degrees in information engineering from the Tohoku University, Sendai, Japan, in 1980, 1982 and 1985, respectively.

From 1988 to 1989 , he was a Visiting Researcher with the Federal German Institute for Geoscience and Natural Resources (BGR), Hannover, Germany. Since 1997, he has been a Professor with Tohoku University, where he was a Distinguished Professor of Tohoku University from 2007 to 2011 and the Director of Center for Northeast Asian Studies from 2009 to 2013. He is a visiting Professor with Jilin University, China; the Delft University of Technology, Delft, The Netherlands; and the Mongolian University of Science and Technology, Ulan Bator, Mongolia. He has conducted the development of Ground Penetrating Radar (GPR) sensors for humanitarian demining and developed a sensor, Advanced Landmine Imaging System, which is a handheld dual sensor that detected more than 80 mines in mine fields in Cambodia. His research interests include transient electromagnetic and antenna, radar polarimetry, GPR, borehole radar, electromagnetic induction sensing, and interferometric and polarimetric SAR.

Mr. Sato was a member of the IEEE GRSS AdCom from 2006 to 2014 He was a recipient of the 2014 Frank Frischknecht Leadership Award from SEG for his contribution to his sustained and important contributions to near-surface geophysics in Ground Penetrating Radar, the IEICE Best paper award (Kiyasu Award) in 2017, the Achievement Award in 2019, IEEE GRSS Education Award in 2012 and IEEE Ulrich L. Rohde Innovative Conference Paper Awards on Antenna Measurements and Applications in 2017. He was the Chair of the IEEE GRSS Japan Chapter from 2006 to 2007. He served as the General Chair for IEEE International Geoscience and Remote Sensing Symposium (IGARSS) 2011 and the Technical Chair of GPR1996. $\mathrm{He}$ is an Associate Editor of IEEE Geoscience AND Remote SENSING LETTERS, and a Guest Editor of the special issue of GPR2006 and GPR2010 in IEEE Transactions on Geoscience and Remote Sensing, and IGARSS2011, GPR2012 and GPR2014 in IEEE JOURNAL OF SELECTED TOPICS IN APPLIED EARTH OBSERVATIONS AND REMOTE SENSING. 